\title{
Predictive Value of Prognostic Nutritional Index on COVID-19 Severity
}

\begin{abstract}
Zhong-hua Wang ${ }^{1 \dagger}$, Ying-Wen Lin ${ }^{2,3+}$, Xue-biao Wei ${ }^{1+}$, Fei Li ${ }^{4}$, Xiao-Long Liao ${ }^{1}$, Hui-qing Yuan ${ }^{5}$, Dao-zheng Huang ${ }^{1}$, Tie-he Qin ${ }^{1 *}$, Heng Geng ${ }^{6 *}$ and Shou-hong Wang ${ }^{1 *}$

${ }^{1}$ Department of Critical Care Medicine, Guangdong Provincial Geriatrics Institute, Guangdong Provincial People's Hospital, Guangdong Academy of Medical Sciences, Guangzhou, China, ${ }^{2}$ Department of Cardiology, Guangdong Cardiovascular Institute, Guangdong Provincial Key Laboratory of Coronary Heart Disease Prevention, Guangdong Provincial People's Hospital, Guangdong Academy of Medical Sciences, Guangzhou, China, ${ }^{3}$ Shantou University Medical College, Shantou, China, ${ }^{4}$ Emergency Department, The First People's Hospital of Jingzhou, Jingzhou, China, ${ }^{5}$ Department of Respiratory and Critical Care Medicine, The First People's Hospital of Shaoguan, Shaoguan, China, ${ }^{6}$ Department of Critical Care Medicine, The First People's Hospital of Jingzhou, Jingzhou, China
\end{abstract}

OPEN ACCESS

Edited by:

Alessio Molfino,

Sapienza University of Rome, Italy

Reviewed by:

Pengfei Rong,

Central South University, China

Caterina Conte,

Università telematica San

Raffaele, Italy

*Correspondence:

Tie-he Qin

qintiehe@163.com

Heng Geng

20781236@qq.com

Shou-hong Wang

gdwangshouhong@163.com

†These authors share first authorship

Specialty section

This article was submitted to

Clinical Nutrition,

a section of the journal

Frontiers in Nutrition

Received: 13 July 2020 Accepted: 17 December 2020

Published: 14 January 2021

Citation:

Wang Z-h, Lin Y-W, Wei X-b, Li F

Liao $X-L$, Yuan $H-q$, Huang $D-Z$,

Qin T-h, Geng H and Wang S-h (2021)

Predictive Value of Prognostic

Nutritional Index on COVID-19

Severity. Front. Nutr. 7:582736.

doi: 10.3389/fnut.2020.582736
Background: The prognostic nutritional index (PNI) has been described as a simple risk-stratified tool for several diseases. We explored the predictive role of the $\mathrm{PNI}$ on coronavirus disease 2019 (COVID-19) severity.

Methods: A total of 101 patients with COVID-19 were included in this retrospective study from January 2020 to March 2020. They were divided into two groups according to COVID-19 severity: non-critical $(n=56)$ and critical $(n=45)$. The PNI was calculated upon hospital admission: $10 \times$ serum albumin $(\mathrm{g} / \mathrm{dL})+0.005 \times$ total lymphocyte count $\left(/ \mathrm{mm}^{3}\right)$. Critical COVID-19 was defined as having one of the following features: respiratory failure necessitating mechanical ventilation; shock; organ dysfunction necessitating admission to the intensive care unit (ICU). The correlation between the PNI with COVID-19 severity was analyzed.

Results: The PNI was significantly lower in critically ill than that in non-critically ill patients $(P<0.001)$. The receiver operating characteristic curve indicated that the PNI was a good discrimination factor for identifying COVID-19 severity $(P<0.001)$. Multivariate logistic regression analysis showed the $\mathrm{PNI}$ to be an independent risk factor for critical illness due to COVID-19 $(P=0.002)$.

Conclusions: The PNI is a valuable biomarker that could be used to discriminate COVID-19 severity.

Keywords: prognostic nutritional index, COVID-19, severity, prognosis, nutrition - clinical

\section{INTRODUCTION}

Coronavirus disease 2019 (COVID-19) is a life-threatening respiratory illness caused by severe acute respiratory syndrome coronavirus (SARS-CoV)-2 infection. On 11 March 2020, the World Health Organization (WHO) declared a pandemic due to COVID-19. SARS-CoV-2 is spreading rapidly worldwide and wreaking havoc in public healthcare systems. More than 65 million cases of SARS-CoV-2 infection have been recorded worldwide, including 1.5 million confirmed deaths up to December 2020, according to the WHO. 
The clinical manifestations of COVID-19 are extremely broad. They range from asymptomatic disease and mild, selflimiting respiratory illness with nonspecific symptoms to severe pneumonia with life-threatening complications, including acute respiratory distress syndrome, multiorgan dysfunction, and death. These complications hamper appropriate care of these patients (1-4). It has been reported that $10-20 \%$ of patients diagnosed with COVID-19 require critical care, which places a huge burden upon healthcare facilities (5). Thus, early identification of patients at risk of becoming critically ill is vitally important to allocate medical resources and to provide early intervention to improve the prognosis. Interestingly, some epidemiological features and clinical characteristics have been identified as risk factors for COVID-19 severity (6). However, a novel, feasible, and readily assessable biomarker that can predict COVID-19 severity is not available.

The immune and nutritional status of the host is associated with susceptibility to and severity of infectious diseases (710). Hypoalbuminemia is present during inflammation and malnutrition, and is associated with poor clinical outcomes in acutely ill patients $(11,12)$. Conversely, white blood cell (WBC) counts in peripheral blood have been reported to reflect local or systemic inflammation (13). Lymphocytopenia is one of the most common clinical manifestations of acute viral infection $(14,15)$. Patients with severe COVID-19 exhibit prominent hypoalbuminemia and lymphocytopenia compared with those with non-severe COVID-19 (16-18). Analyses of molecular and immunological data from 326 patients with COVID19 in Shanghai (China) revealed that host factors (especially lymphocytopenia) could predict disease progression (19).

The prognostic nutritional index (PNI) (20) is an objective assessment index reflecting the immune-nutritional status of patients. The PNI is calculated by multiplication of the serum albumin level and lymphocyte count. It is a valuable screening tool for patient prognosis in several diseases (21-24). Poor nutritional status and immune dysfunction (especially depletion of $\mathrm{T}$ lymphocytes) have been considered to be risk factors for severe infection by SARS-CoV-2 (25). However, the importance of this immune-nutritional index in terms of predicting COVID-19 severity has yet to be elucidated.

We explored the association of the PNI with COVID19 severity and assessed its prognostic value using multivariate models.

\section{MATERIALS AND METHODS}

\section{Ethical Approval of the Study Protocol}

The study protocol was approved by the Ethics Committee of The First People's Hospital of Jingzhou (L20200208) in Jingzhou, China. The requirement for written informed consent was waived due to the retrospective design of the study.

\section{Study Population}

This observational retrospective study involved 101 patients with laboratory-confirmed SARS-CoV-2 infection at The First People's Hospital of Jingzhou (Jingzhou, China) from January
2020 to March 2020. A laboratory-confirmed case of COVID19 was based on the result of real-time reverse transcriptionquantitative polymerase chain reaction of a nasopharyngeal swab, in accordance with WHO guidelines (26). COVID-19 severity upon hospital admission was defined based on the fifth version of the National Health Commission Guideline on the Management of Novel Coronavirus Pneumonia. Patients were categorized into "critical" and "non-critical" COVID-19 groups. COVID19 patients who were critically ill were identified if they had one of the following features: respiratory failure necessitating mechanical ventilation; shock; organ dysfunction necessitating admission to the intensive care unit (ICU).

\section{Data Collection}

Demographic and clinical data were obtained retrospectively from electronic medical records in The First People's Hospital of Jingzhou, and assimilated by three researchers (Wang, Li, and Geng). Data were transferred to other team members in Guangdong Provincial People's Hospital (Guangzhou, China) for statistical analyses. The electronic medical records, nursing records, laboratory results, and imaging findings of all enrolled patients were reviewed. Data on age, sex, comorbidities, treatment plans, and duration of hospital stay were extracted. Laboratory results [complete blood count, hematology, coagulation testing, liver/renal function, as well as levels of electrolytes, C-reactive protein (CRP), procalcitonin, lactate dehydrogenase, and creatine kinase] were collected from the first electronic medical record after hospital admission. The PNI was calculated according to the following formula (20): PNI $=10 \times$ serum albumin $(\mathrm{g} / \mathrm{dL})+0.005 \times$ peripheral lymphocyte count $\left(/ \mathrm{mm}^{3}\right)$. The Glasgow Prognostic Score (GPS) was obtained based on the level of CRP and albumin (27).

\section{Statistical Analyses}

Statistical analyses were undertaken using SPSS 24.0 (IBM, Armonk, NY, USA). Continuous variables are expressed as the mean \pm standard deviation (SD), median values, interquartile ranges or simple ranges. Categorical variables are summarized as counts and percentages. Continuous data with a normal distribution were compared using the Student's $t$-test. Data with a non-normal distribution were compared using the Wilcoxon rank-sum test and presented as the median and interquartile range. Categorical data were compared using the chi-square test or Fisher's exact test. Baseline data including demographic, history of illness, clinical and laboratory variables were included in the logistic analyses to assess their associations with COVID19 severity. Univariate and multivariate logistic regression analyses were carried out to determine the risk factors for severe COVID-19. The PNI and variables with $P<0.05$ in the univariate logistic regression analysis were included in the multivariate logistic regression analysis to evaluate COVID19 severity. Then, the adjusted odds ratio (OR) and 95\% confidence interval (CI) were calculated. The optimal cutoff of the PNI for predicting COVID-19 severity was determined by analyses of the receiver operating characteristic (ROC) curve. We also calculate the net reclassification improvement (NRI) and integrated discrimination improvement (IDI) to compare 
the discrimination ability between different prediction models. $P<0.05$ was considered significant.

\section{RESULTS}

\section{Patient Characteristics at Baseline}

A total of 101 patients with laboratory-confirmed COVID-19 were included in this study. Their demographic and clinical characteristics are listed in Table 1. The mean age of the study cohort was $56 \pm 17$ years. The study cohort comprised $47 / 101$ (46.5\%) males.

Among 101 patients with COVID-19, 45 (44.6\%) were categorized into the critical group and 56 (55.4\%) into the noncritical group. The mean age of the latter was $49.3 \pm 16.9$ years, whereas that of the critical group was $64.9 \pm 13.2$ years.

Patients critically ill with COVID-19 had a significantly higher risk of in-hospital death than those who were not critically ill with COVID-19 (13.3\% vs. $0, P=0.017)$. Compared with

TABLE 1 | Clinical characteristics stratified by the severity of COVID-19.

\begin{tabular}{|c|c|c|c|}
\hline & Non-critical $(n=56)$ & Critical $(n=45)$ & $P$-value \\
\hline Age (years) & $49.3 \pm 16.9$ & $64.9 \pm 13.2$ & $<0.001$ \\
\hline \multicolumn{4}{|l|}{ SEX, $N(\%)$} \\
\hline Male & 22 (39.3) & $25(55.6)$ & 0.103 \\
\hline Female & $34(60.7)$ & $20(44.4)$ & \\
\hline Hypertension, $n$ (\%) & $13(23.2)$ & 15 (33.3) & 0.259 \\
\hline Diabetes, $n(\%)$ & $2(3.6)$ & $2(4.4)$ & 1.000 \\
\hline Heart rate, bpm & $89.9 \pm 15.3$ & $90.0 \pm 17.7$ & 0.976 \\
\hline MAP, mmHg & $97.0 \pm 11.9$ & $98.4 \pm 19.3$ & 0.693 \\
\hline CRP (mg/L) & $7.4(1.4,24.5)$ & $20.2(7.2,55.1)$ & 0.002 \\
\hline $\mathrm{SCr}(\mu \mathrm{mol} / \mathrm{L})$ & $60.1(51.0,71.3)$ & $73.9(57.0,86.2)$ & 0.036 \\
\hline WBC count, $\times 10^{9} / \mathrm{L}$ & $4.9(3.7,5.7)$ & $6.1(4.3,8.6)$ & 0.009 \\
\hline Neutrophil count, $\times 10^{9} / \mathrm{L}$ & $3.1(2.2,3.9)$ & $4.0(2.6,7.3)$ & 0.004 \\
\hline Lymphocyte count, $\times 10^{9} / \mathrm{L}$ & $1.3(0.9,1.6)$ & $1.0(0.6,1.3)$ & 0.005 \\
\hline Platelet count, $\times 10^{9} / \mathrm{L}$ & $164.2 \pm 68.1$ & $172.0 \pm 81.3$ & 0.602 \\
\hline Hemoglobin (g/L) & $121.8 \pm 14.1$ & $114.0 \pm 21.9$ & 0.041 \\
\hline \multicolumn{4}{|l|}{ LIVER FUNCTION TESTS } \\
\hline ALT (U/L) & $13.5(9.3,24.8)$ & $26.0(14.5,48.0)$ & $<0.001$ \\
\hline Albumin (g/L) & $41.4 \pm 3.6$ & $37.4 \pm 4.3$ & $<0.001$ \\
\hline TBIL, $\mu \mathrm{mol} / \mathrm{L}$ & $11.2(9.2,13.3)$ & $11.9(9.4,17.2)$ & 0.367 \\
\hline DBIL, $\mu \mathrm{mol} / \mathrm{L}$ & $3.7(3.0,4.8)$ & $4.5(3.2,6.4)$ & 0.059 \\
\hline CK, U/L & $63.0(50.5,88.5)$ & $64.0(32.5,146.5)$ & 0.874 \\
\hline CK-MB, U/L & $12.0(9.5,15.0)$ & $14.0(9.3,17.0)$ & 0.252 \\
\hline $\mathrm{PNI}$ & $48.0 \pm 4.5$ & $42.4 \pm 5.4$ & $<0.001$ \\
\hline \multicolumn{4}{|l|}{ TREATMENT } \\
\hline Antibiotic & $55(98.2)$ & $31(68.9)$ & $<0.001$ \\
\hline Glucocorticoid & $22(39.3)$ & $29(64.4)$ & 0.012 \\
\hline Interferon & $13(23.2)$ & 24 (53.3) & 0.002 \\
\hline In-hospital death & 0 & 6 (13.3) & 0.017 \\
\hline
\end{tabular}

MAP, mean arterial pressure; CRP, C-reactive protein; SCr, serum creatinine; WBC, white blood cell; ALT, alanine transaminase; TBIL, total bilirubin; DBIL, direct bilirubin; CK, creatine kinase; CK-MB, creatine kinase-MB; PNI, prognostic nutritional index; MACES, major adverse clinical events, defined by death, stroke, and requirement of dialysis. the non-critical group, patients in the critical group had a higher CRP level, higher WBC count, higher level of alanine aminotransferase (ALT), and lower hemoglobin level. Moreover, the PNI was significantly lower in the critical group as compared with that in the non-critical group $(42.4 \pm 5.4$ vs. $48.0 \pm 4.5$, $P<0.001)$.

\section{Association of the PNI With COVID-19 Severity}

Univariate logistic regression analysis showed that the PNI was associated with critical illness due to COVID-19 (odds ratio $(\mathrm{OR})=0.80, P<0.001$ ) (Table 2). The components of the PNI (serum level of albumin, and lymphocyte count) were strongly associated with critical illness due to COVID-19. Additional significant indicators were age, CRP level, estimated glomerular filtration rate $<90 \mathrm{~mL} / \mathrm{min} / 1.73 \mathrm{~m}^{2}$, WBC count, anemia, and ALT level. After adjustment of these variables, multivariate regression analysis showed that the serum albumin level and lymphocyte count were independent predictors for critical illness due to COVID-19. When incorporated into multivariate analysis in model 2, the PNI remained an independent predictor for critical illness due to COVID-19 (OR $=0.81$, 95\%CI: 0.71-0.92, $P=0.002$ ) (Table 3), indicating that the risk of critical COVID19 would decrease by $19 \%$ with every unit increase of PNI. In addition, age was found to be independently associated with severe COVID-19 (OR 1.05, 95\%CI: 1.01-1.10, $P=0.022$ ).

TABLE 2 | Univariate logistic regression analysis for the severity of COVID-19.

\begin{tabular}{|c|c|c|c|}
\hline Clinical variables & OR & $95 \% \mathrm{Cl}$ & $p$-value \\
\hline $\mathrm{PNI}$ & 0.80 & $0.73,0.88$ & $<0.001$ \\
\hline Age & 1.07 & $1.04,1.10$ & $<0.001$ \\
\hline Female sex & 0.52 & $0.23,1.15$ & 0.105 \\
\hline Hypertension & 1.65 & $0.69,3.98$ & 0.261 \\
\hline Diabetes & 1.26 & $0.17,9.28$ & 0.823 \\
\hline Heart rate & 1.00 & $0.98,1.03$ & 0.976 \\
\hline MAP & 1.01 & $0.98,1.03$ & 0.676 \\
\hline CRP & 1.02 & $1.00,1.03$ & 0.011 \\
\hline eGFR $<90 \mathrm{ml} / \mathrm{min} / 1.73 \mathrm{~m}^{2}$ & 3.94 & $1.37,11.39$ & 0.011 \\
\hline WBC count & 1.21 & $1.06,1.39$ & 0.006 \\
\hline Lymphocyte count & 0.29 & $0.12,0.69$ & 0.005 \\
\hline Platelet count & 1.00 & $1.00,1.01$ & 0.598 \\
\hline Anemia & 2.86 & $1.25,6.51$ & 0.013 \\
\hline Albumin & 0.78 & $0.69,0.88$ & $<0.001$ \\
\hline ALT & 1.02 & $1.00,1.03$ & 0.029 \\
\hline TBIL & 1.05 & $0.98,1.13$ & 0.158 \\
\hline DBIL & 1.18 & $1.00,1.39$ & 0.052 \\
\hline CK & 1.01 & $1.00,1.01$ & 0.069 \\
\hline CK-MB & 1.02 & $0.98,1.07$ & 0.319 \\
\hline
\end{tabular}

OR, odds ratio; $C l$, confidence interval; PNI, prognostic nutritional index; MAP, mean arterial pressure; CRP, C-reactive protein; eGFR, estimated glomerular filtration rate; WBC, white blood cell; $A L T$, alanine transaminase; TBIL, total bilirubin; DBIL, direct bilirubin; CK, creatine kinase; $C K-M B$, creatine kinase-MB. 
TABLE 3 | Multivariate logistic regression analysis for the severity of COVID-19.

\begin{tabular}{|c|c|c|c|}
\hline Clinical variables & OR & $95 \% \mathrm{Cl}$ & $p$-value \\
\hline \multicolumn{4}{|l|}{ MODEL 1} \\
\hline Age & 1.05 & $1.01,1.10$ & 0.019 \\
\hline CRP & 0.99 & $0.97,1.01$ & 0.134 \\
\hline eGFR $<90 \mathrm{ml} / \mathrm{min} / 1.73 \mathrm{~m}^{2}$ & 2.36 & $0.54,10.35$ & 0.254 \\
\hline WBC count & 1.34 & $1.01,1.79$ & 0.046 \\
\hline Anemia & 1.04 & $0.30,3.67$ & 0.947 \\
\hline ALT & 1.01 & $0.99,1.03$ & 0.33 \\
\hline Lymphocyte count & 0.21 & $0.06,0.73$ & 0.014 \\
\hline Albumin & 0.84 & $0.72,0.98$ & 0.022 \\
\hline \multicolumn{4}{|l|}{ MODEL 2} \\
\hline Age & 0.81 & $0.71,0.92$ & 0.002 \\
\hline CRP & 0.99 & $0.97,1.01$ & 0.213 \\
\hline eGFR $<90 \mathrm{ml} / \mathrm{min} / 1.73 \mathrm{~m}^{2}$ & 2.46 & $0.57,10.58$ & 0.227 \\
\hline WBC count & 1.26 & $0.98,1.61$ & 0.071 \\
\hline Anemia & 0.98 & $0.29,3.38$ & 0.978 \\
\hline ALT & 1.01 & $0.99,1.03$ & 0.195 \\
\hline PNI & 0.81 & $0.71,0.92$ & 0.002 \\
\hline
\end{tabular}

OR, odds ratio; $\mathrm{Cl}$, confidence interval; CRP, C-reactive protein; eGFR, estimated glomerular filtration rate; WBC, white blood cell; ALT, alanine transaminase; PNI, prognostic nutritional index.

\section{Comparison of the PNI and Its Components With Other Prognostic Factors}

We explored the ability of the PNI and each of its components (serum level of albumin and lymphocyte count) using the area under the ROC curve (AUC) for the prediction of critical illness due to COVID-19.

The AUC of the PNI, serum level of albumin, and lymphocyte count was 0.790 (95\%CI: 0.701-0.880), 0.757 (0.662-0.851), and $0.663(0.556-0.769)$, respectively (Figure 1). The AUC of the PNI was significantly higher than that of the lymphocyte count but not the serum level of albumin. However, addition of the lymphocyte count to the serum level of albumin improved the prediction of COVID-19 severity (NRI $=0.4848,95 \%$ CI: $0.1094-$ 0.8604, $P=0.011$; IDI $=0.0464,95 \% \mathrm{CI}: 0.0086-0.0844, P=$ 0.016). A PNI $<43$ was the optimal threshold for predicting severe COVID-19, had a sensitivity of $85.7 \%$ and specificity of $60.0 \%$, and showed significantly higher accuracy than GPS for predicting critical illness due to COVID-19 (AUC: 0.789 vs. 0.692, $P=0.028)$ (Figure 2).

\section{PNI and Other Clinical Parameters}

The clinical characteristics of patients with COVID-19 stratified by the cutoff of the PNI level at the time of hospital admission are shown in Table 4. Patients with a PNI $<43$ were older with lower mean arterial pressure, higher level of CRP, higher level of creatine kinase- $\mathrm{MB}$, and lower hemoglobin level. The prevalence of critical illness was significantly higher in patients with a lower PNI than that in patients with a higher PNI. Linear regression analysis showed that the PNI in patients with COVID-19 was negatively correlated with the CRP level and positively correlated with the hemoglobin level $(r=-0.454, P<0.001 ; r=0.332$, $P<0.001$, respectively) (Figure 3).

\section{DISCUSSION}

The present study demonstrated the PNI to be an independent predictor of COVID-19 severity. A PNI $<43$ was the optimal cutoff for risk assessment. Risk factors (e.g., older age and PNI $<43$ ) could be used to identify potential critically-ill COVID19 patients at an early stage. As compared with the GPS (which includes the CRP level and lymphocyte count), the PNI had significantly higher accuracy in predicting critical illness due to COVID-19.

The Chinese Center for Disease Control and Prevention reported that $81 \%$ of patients with COVID- 19 have mild disease, $14 \%$ have severe disease, and 5\% progress to critical illness with organ failure; thus, the mortality rate in the critically-ill group is $\leq 49 \%$ (28). Moreover, the interval from the initial development of symptoms to the onset of critical illness is 10 days (29). The delayed onset of critical illness in patients with COVID-19 and high mortality rate have prompted an urgent search for a biomarker that can allow an early diagnosis.

Herein, we found that older age was a risk factor for critical illness for COVID-19 patients, a finding that is consistent with the results of other studies $(16,30,31)$. We also explored the predictive role of the PNI: it was independently associated with the severity of COVID-19 and a poor prognosis. The PNI is calculated based on the serum level of albumin and peripheral lymphocyte count. It was first conceptualized and applied by Buzby and colleagues to estimate the operative risk in gastrointestinal surgery (20).

Albumin is a well-known plasma protein with multiple physiological functions; the level of this protein fluctuates during inflammation and malnutrition (12, 32). Hypoalbuminemia has been strongly associated with poor clinical outcomes in acutely ill patients (11). Recent observational studies on patients with COVID-19 have demonstrated that a reduced albumin concentration in serum is associated with increased disease severity (17). Furthermore, the reduced production and increased loss of albumin in serum can be seen in patients with damage to liver function and renal function, which are common attributes in critically ill COVID-19 patients (33). In the current study, the PNI was negatively correlated with the CRP level. Interestingly, an inflammatory status promotes albumin degradation and a reduction in its level in the liver $(34,35)$; severe inflammation is associated with a progressively lower serum albumin level (34). Accumulating evidence has suggested that a subgroup of patients with severe COVID-19 exhibit a hyperinflammatory status with a "cytokine storm," which further supports the potential predictive role of hypoalbuminemia $(2,36$, 37).

Lymphocytopenia has been shown to be associated with increased severity of COVID-19 (38). Patients who died from COVID-19 were reported to have a significantly lower lymphocyte count than that of survivors $(33,37)$. A dramatically 


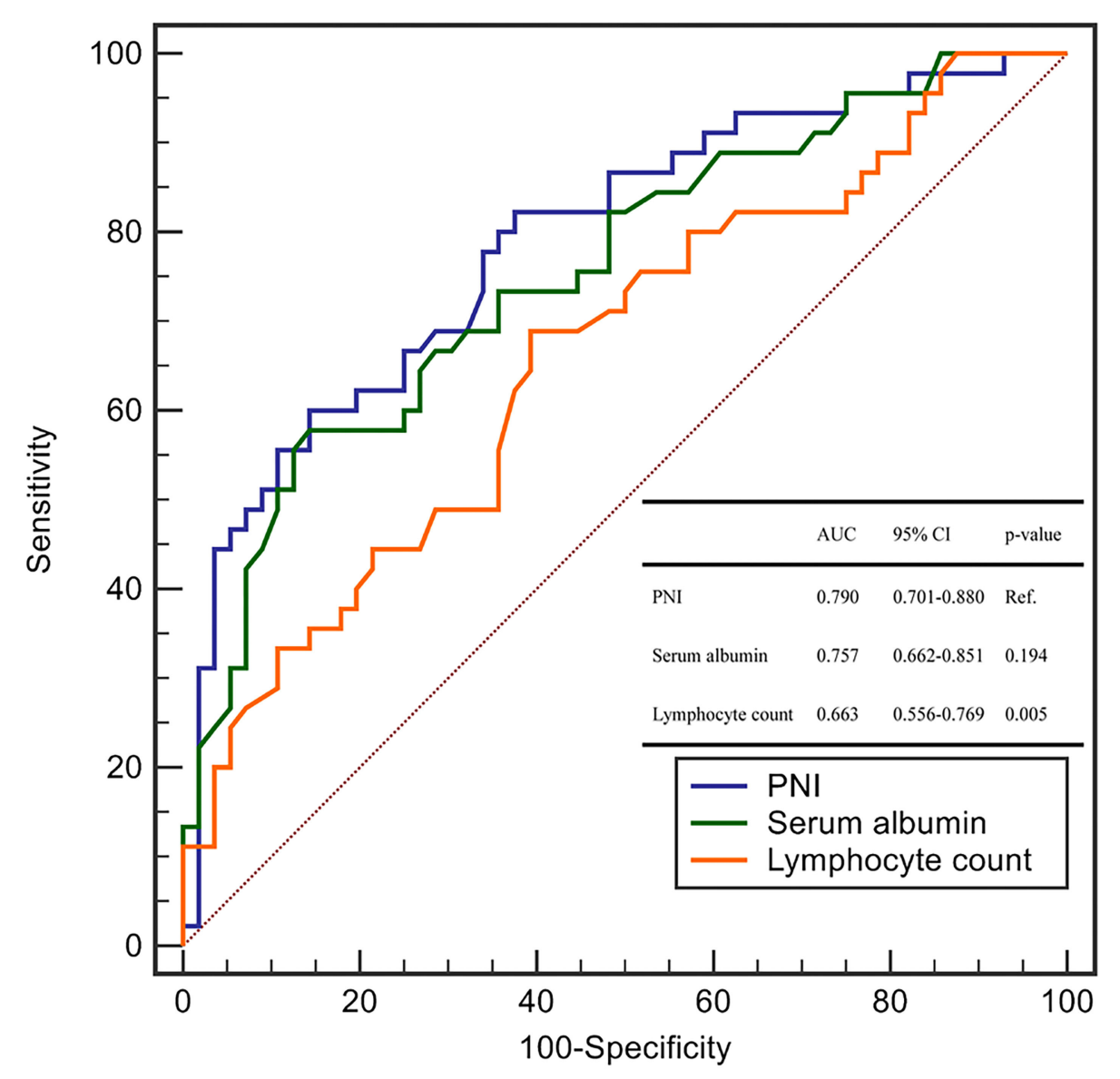

FIGURE 1 | The ROC curves of PNI and its components for the prediction of critical COVID-19.

reduced number of cluster of differentiation (CD)4+ $\mathrm{T}$ cells, $\mathrm{CD} 8+\mathrm{T}$ cells, $\mathrm{B}$ cells, and natural killer (NK) cells has been noted in patients with severe COVID-19 $(17,39)$. Expression of NKG2A (a marker of the exhausted function of NK cells and CD8+ $\mathrm{T}$ cells) is increased significantly in COVID19 patients (40). In addition, restoration of the lymphocyte count from peripheral blood has been seen in patients with viral clearance (41). The potential compounding immunological insults caused by SARS-CoV-2 are major mechanisms of COVID-19 progression, which suggests that surveillance of the lymphocyte count is valuable in the early screening of critical illness due to COVID-19 (42). Intriguingly, treatments that address the immunopathology of SARS-CoV-2 are under intensive focus currently (43).

Taken together, it can be deduced that the PNI characterizes the immune and inflammatory status in patients with COVID19. We validated, for the first time, the PNI as a useful biomarker that is independently associated with COVID-19 severity. We also compared the discrimination ability of the PNI with its components. The PNI conferred a significantly higher predictive value for COVID-19 severity than that by the lymphocyte count. However, when compared with the serum level of albumin, the PNI had a higher AUC for prediction of COVID-19 severity but was not significant using the Concordance Index (C-Index). However, analyses of the NRI and IDI demonstrated that the PNI (which contains the albumin level and lymphocyte count) conferred additional performance for the prediction of critical illness due to COVID-19 as compared with that of albumin level alone. Although the CIndex and AUC are the most popular metrics for evaluating the discrimination performance of prognostic models, both have limitations. Once the C-Index or AUC reach a certain level, they require large effect sizes from the newly added markers to obtain a noticeable increase, but such large effect sizes are uncommon and unrealistic. The NRI is a simple (but effective) approach for comparing the discrimination ability between two models, especially for the improvement conferred by inclusion of new variables (44).

The present study had three main limitations. First, nearly half of enrolled patients in the study had critical illness due to COVID-19. This high proportion of critically ill patients in the study cohort could have been because 


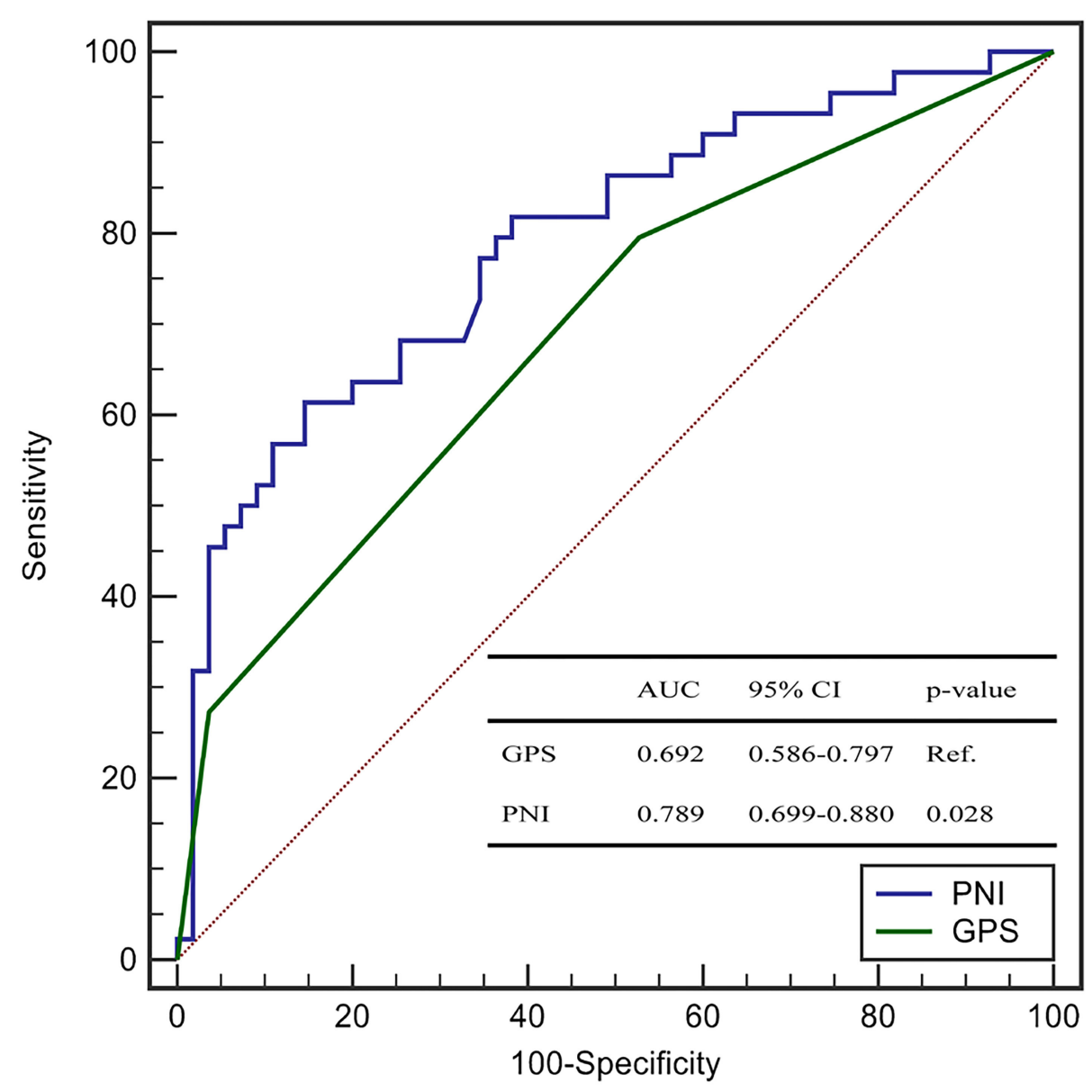

FIGURE 2 | The ROC curves of PNI and GPS for the prediction of critical COVID-19.
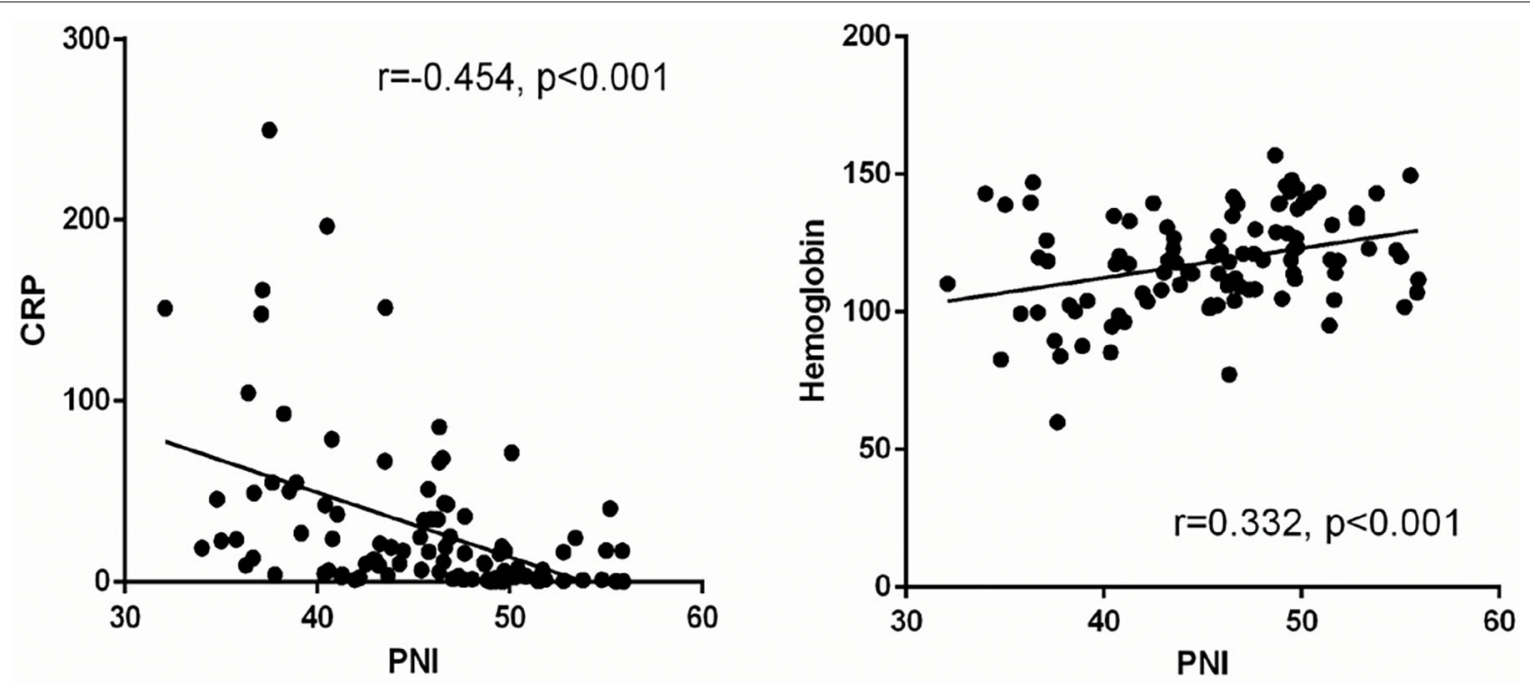

FIGURE 3 | Linear regression between PNI and clinical parameters (CRP and hemoglobin). 
TABLE 4 | Clinical characteristics stratified by PNI level at admission.

\begin{tabular}{|c|c|c|c|}
\hline & $\mathrm{PNI} \geq 43(n=69)$ & $\mathrm{PNI}<43(n=32)$ & $P$-value \\
\hline Age (years) & $52.6 \pm 17.4$ & $64.3 \pm 13.7$ & $<0.001$ \\
\hline \multicolumn{4}{|l|}{ SEX, $N(\%)$} \\
\hline Male & $33(47.8)$ & $14(43.8)$ & 0.702 \\
\hline Female & $36(52.2)$ & $18(56.3)$ & \\
\hline Hypertension, $n$ (\%) & $17(24.6)$ & $11(34.4)$ & 0.309 \\
\hline Diabetes, $n(\%)$ & $2(2.9)$ & $2(6.3)$ & 0.799 \\
\hline Heart rate, bpm & $88.6 \pm 15.0$ & $93.0 \pm 18.7$ & 0.213 \\
\hline Mean arterial pressure, $\mathrm{mmHg}$ & $100.7 \pm 13.5$ & $91.1 \pm 17.8$ & 0.003 \\
\hline C reactive protein (mg/L) & $10.3(1.5,24.5)$ & $25.4(7.1,73.1)$ & 0.001 \\
\hline Serum creatinine $(\mu \mathrm{mol} / \mathrm{L})$ & $63.4(54.3,80.4)$ & $62.7(48.6,84.7)$ & 0.506 \\
\hline White blood cell count, $\times 10^{9} / \mathrm{L}$ & $5.1(3.9,6.6)$ & $5.8(4.1,10.9)$ & 0.116 \\
\hline Neutrophil count, $\times 10^{9} / \mathrm{L}$ & $3.2(2.3,4.1)$ & $4.6(2.6,9.3)$ & 0.013 \\
\hline Lymphocyte count, $\times 10^{9} / \mathrm{L}$ & $1.3(1.0,1.6)$ & $0.8(0.5,1.0)$ & $<0.001$ \\
\hline Platelet count, $\times 10^{9} / \mathrm{L}$ & $163.9 \pm 71.1$ & $175.7 \pm 80.3$ & 0.459 \\
\hline Hemoglobin (g/L) & $122.6 \pm 15.2$ & $109.2 \pm 21.2$ & 0.002 \\
\hline \multicolumn{4}{|l|}{ LIVER FUNCTION TESTS } \\
\hline ALT (U/L) & $16.0(11.0,27.0)$ & $26.5(13.0,66.5)$ & 0.082 \\
\hline Albumin (g/L) & $42.0 \pm 2.7$ & $34.6 \pm 2.6$ & $<0.001$ \\
\hline TBIL, $\mu \mathrm{mol} / \mathrm{L}$ & $11.5(9.5,13.6)$ & $11.9(9.1,18.5)$ & 0.499 \\
\hline DBIL, $\mu \mathrm{mol} / \mathrm{L}$ & $3.9(3.0,4.7)$ & $5.0(3.2,7.0)$ & 0.049 \\
\hline Creatine kinase, U/L & $67.0(50.3,97.0)$ & $51.0(31.3,113.5)$ & 0.241 \\
\hline Creatine kinase-MB, U/L & $11.0(9.0,15.0)$ & $15.0(11.0,22.0)$ & 0.005 \\
\hline Critical cases, $n(\%)$ & $20(29.0)$ & $25(78.1)$ & $<0.001$ \\
\hline \multicolumn{4}{|l|}{ TREATMENT } \\
\hline Antibiotic therapy & $61(88.4)$ & $25(78.1)$ & 0.293 \\
\hline Glucocorticoid therapy & $33(47.8)$ & $18(56.3)$ & 0.431 \\
\hline Interferon therapy & $20(29.0)$ & $17(53.1)$ & 0.019 \\
\hline Length of stay, days & $25(20,30)$ & $26(19,35)$ & 0.461 \\
\hline In-hospital death & $2(2.9)$ & $4(12.5)$ & 0.148 \\
\hline
\end{tabular}

$O R$, odds ratio; $C l$, confidence interval; PNI, prognostic nutritional index; $A L T$, alanine transaminase; TBIL, total bilirubin; DBIL, direct bilirubin.

our hospital was the main facility in Jingzhou responsible for admitting and caring for critically ill patients with COVID-19. Second, due to the retrospective design and the urgency of the epidemics, anthropometrics data is lacking and factors such as levels of interleukin-6 and ferritin were not measured, so their role in predicting COVID-19 severity could not be considered during data analyses. Third, this was a single-center study with a small study cohort and small number of critically ill cases, which would have underpowered our analyses.

\section{REFERENCES}

1. Weiss P, Murdoch DR. Clinical course and mortality risk of severe COVID-19. Lancet Lond Engl. (2020) 395:1014-5. doi: 10.1016/S0140-6736(20)30633-4

2. Huang C, Wang Y, Li X, Ren L, Zhao J, Hu Y, et al. Clinical features of patients infected with 2019 novel coronavirus in Wuhan, China. Lancet Lond Engl. (2020) 395:497-506. doi: 10.1016/S0140-6736(20)30183-5

\section{CONCLUSIONS}

The PNI is a valuable and inexpensive biomarker that is independently associated with COVID-19 severity. Patients with a PNI $<43$ were likely to be critically ill with COVID-19. Our findings could help clinicians to identify patients at a risk of critical illness at an early stage of COVID-19 and who have a poor prognosis.

\section{DATA AVAILABILITY STATEMENT}

The original contributions presented in the study are included in the article/supplementary material, further inquiries can be directed to the corresponding author/s.

\section{ETHICS STATEMENT}

The studies involving human participants were reviewed and approved by the Ethics Committee of The First People's Hospital of Jingzhou (L20200208). Written informed consent for participation was not required for this study in accordance with the national legislation and the institutional requirements.

\section{AUTHOR CONTRIBUTIONS}

S-hW and HG contributed to the conception or design of the study. Z-hW, FL, Y-WL, X-bW, X-LL, H-qY, and D-zH contributed to the acquisition, analyses, or interpretation of data. Z-hW, Y-WL, and X-bW drafted the manuscript. S-hW and T-hQ revised the manuscript critically. All authors agree to be accountable for all aspects of work to ensure its integrity and accuracy. All authors contributed to the article and approved the submitted version.

\section{FUNDING}

This study was supported by grants from Medical Science and Technology Research Funding of Guangdong (A2019409), the Fundamental Research Funds for the Central Universities (2019MS136), Science and Technology Projects of Guangzhou (201704020124 and 201903010097), and Guangdong Provincial Key Laboratory of Coronary Heart Disease Prevention (2017B030314041).

\section{ACKNOWLEDGMENTS}

We are grateful for the statistical assistance provided by Guozhi Jiang from the School of Public Health within Sun Yat-sen University (Shenzhen, China).

3. Wang D, Hu B, Hu C, Zhu F, Liu X, Zhang J, et al. Clinical characteristics of 138 hospitalized patients with 2019 novel coronavirus-infected pneumonia in Wuhan, China. JAMA. (2020) 323:1061-9. doi: 10.1001/jama.2020.1585

4. Chen N, Zhou M, Dong X, Qu J, Gong F, Han Y, et al. Epidemiological and clinical characteristics of 99 cases of 2019 novel coronavirus pneumonia in Wuhan, China: a descriptive study. Lancet Lond Engl. (2020) 395:50713. doi: $10.1016 / \mathrm{S} 0140-6736(20) 30211-7$ 
5. Rodriguez-Morales AJ, Cardona-Ospina JA, Gutiérrez-Ocampo E, VillamizarPeña R, Holguin-Rivera Y, Escalera-Antezana JP, et al. Clinical, laboratory and imaging features of COVID-19: a systematic review and metaanalysis. Travel Med Infect Dis. (2020) 34:101623. doi: 10.1016/j.tmaid. 2020.101623

6. Liang W, Liang H, Ou L, Chen B, Chen A, Li C, et al. Development and validation of a clinical risk score to predict the occurrence of critical illness in hospitalized patients with COVID-19. JAMA Intern Med. (2020) 180:10819. doi: 10.1001/jamainternmed.2020.2033

7. Beck MA, Levander OA. Host nutritional status and its effect on a viral pathogen. J Infect Dis. (2000) 182 (Suppl. 1):S93-6. doi: 10.1086/ 315918

8. Weger-Lucarelli J, Carrau L, Levi LI, Rezelj V, Vallet T, Blanc H, et al. Host nutritional status affects alphavirus virulence, transmission, and evolution. PLoS Pathog. (2019) 15:e1008089. doi: 10.1371/journal.ppat. 1008089

9. Chen X, Liu S, Goraya MU, Maarouf M, Huang S, Chen J-L. Host immune response to influenza A virus infection. Front Immunol. (2018) 9:320. doi: 10.3389/fimmu.2018.00320

10. Zheng J, Perlman S. Immune responses in influenza A virus and human coronavirus infections: an ongoing battle between the virus and host. Curr Opin Virol. (2018) 28:43-52. doi: 10.1016/j.coviro. 2017.11.002

11. Vincent J-L, Dubois M-J, Navickis RJ, Wilkes MM. Hypoalbuminemia in acute illness: is there a rationale for intervention?: A meta-analysis of cohort studies and controlled trials. Ann Surg. (2003) 237:31934. doi: 10.1097/01.SLA.0000055547.93484.87

12. Don BR, Kaysen G. Serum albumin: relationship to inflammation and nutrition. Semin Dial. (2004) 17:432-7. doi: 10.1111/j.0894-0959.2004. 17603.x

13. Wang SC, Chou JF, Strong VE, Brennan MF, Capanu M, Coit DG. Pretreatment neutrophil to lymphocyte ratio independently predicts disease-specific survival in resectable gastroesophageal junction and gastric adenocarcinoma. Ann Surg. (2016) 263:2927. doi: 10.1097/SLA.0000000000001189

14. Cheng Y, Zhao H, Song P, Zhang Z, Chen J, Zhou Y-H. Dynamic changes of lymphocyte counts in adult patients with severe pandemic H1N1 influenza A. J Infect Public Health. (2019) 12:878-83. doi: 10.1016/j.jiph. 2019.05.017

15. Chan PKS, Chen GG. Mechanisms of lymphocyte loss in SARS coronavirus infection. Hong Kong Med J Xianggang Yi Xue Za Zhi. (2008) 14:21-6. Available online at: https://www.hkmj.org/abstracts/v14n4s4/21.htm

16. Guan W-J, Ni Z-Y, Hu Y, Liang W-H, Ou C-Q, He J-X, et al. Clinical characteristics of coronavirus disease 2019 in China. N Engl J Med. (2020) 382:1708-20. doi: 10.1101/2020.02.06.20020974

17. Feng Y, Ling Y, Bai T, Xie Y, Huang J, Li J, et al. COVID-19 with different severity: a multi-center study of clinical features. Am J Respir Crit Care Med. (2020) 201:1380-8. doi: 10.1164/rccm.202002-0445OC

18. Wang Z, Yang B, Li Q, Wen L, Zhang R. Clinical features of 69 cases with coronavirus disease 2019 in Wuhan, China. Clin Infect Dis. (2020) 71:76977. doi: $10.1093 / \mathrm{cid} / \mathrm{ciaa} 272$

19. Zhang X, Tan Y, Ling Y, Lu G, Liu F, Yi Z, et al. Viral and host factors related to the clinical outcome of COVID-19. Nature. (2020) 583:43740. doi: 10.1038/s41586-020-2355-0

20. Buzby GP, Mullen JL, Matthews DC, Hobbs CL, Rosato EF. Prognostic nutritional index in gastrointestinal surgery. Am J Surg. (1980) 139:1607. doi: 10.1016/0002-9610(80)90246-9

21. Correa-Rodríguez M, Pocovi-Gerardino G, Callejas-Rubio J-L, Fernández R, Martín-Amada M, Cruz-Caparros M-G, et al. The prognostic nutritional index and nutritional risk index are associated with disease activity in patients with systemic lupus erythematosus. Nutrients. (2019) 11:638. doi: 10.3390/nu11030638

22. Yang Y, Gao P, Song Y, Sun J, Chen X, Zhao J, et al. The prognostic nutritional index is a predictive indicator of prognosis and postoperative complications in gastric cancer: A meta-analysis. Eur J Surg Oncol. (2016) 42:1176-82. doi: 10.1016/j.ejso.2016.05.029

23. Noh GT, Han J, Cho MS, Hur H, Min BS, Lee KY, et al. Impact of the prognostic nutritional index on the recovery and long-term oncologic outcome of patients with colorectal cancer. J Cancer Res Clin Oncol. (2017) 143:1235-42. doi: 10.1007/s00432-017-2366-x

24. Mirili C, Yilmaz A, Demirkan S, Bilici M, Basol Tekin S. Clinical significance of prognostic nutritional index (PNI) in malignant melanoma. Int J Clin Oncol. (2019) 24:1301-10. doi: 10.1007/s10147-019-01461-7

25. Zabetakis I, Lordan R, Norton C, Tsoupras A. COVID-19: the inflammation link and the role of nutrition in potential mitigation. Nutrients. (2020) 12:1466. doi: 10.3390/nu12051466

26. WHO. Clinical Management of Severe Acute Respiratory Infection When COVID-19 Disease is Suspected. (2020). Available online at: https://www.who. int/publications-detail/clinical-management-of-severe-acute-respiratoryinfection-when-novel-coronavirus-(ncov)-infection-is-suspected (accessed February 28, 2020).

27. Forrest LM, McMillan DC, McArdle CS, Angerson WJ, Dunlop DJ. Evaluation of cumulative prognostic scores based on the systemic inflammatory response in patients with inoperable non-small-cell lung cancer. Br J Cancer. (2003) 89:1028-30. doi: 10.1038/sj.bjc.6601242

28. Wu Z, McGoogan JM. Characteristics of and important lessons from the coronavirus disease 2019 (COVID-19) outbreak in China: summary of a report of 72314 cases from the Chinese Center for Disease Control and Prevention. JAMA. (2020) 323:1239-42. doi: 10.1001/jama. 2020.2648

29. Zhou F, Yu T, Du R, Fan G, Liu Y, Liu Z, et al. Clinical course and risk factors for mortality of adult inpatients with COVID-19 in Wuhan, China: a retrospective cohort study. Lancet Lond Engl. (2020) 395:105462. doi: 10.1016/S0140-6736(20)30566-3

30. Grasselli G, Zangrillo A, Zanella A, Antonelli M, Cabrini L, Castelli A, et al. Baseline characteristics and outcomes of 1591 patients infected with SARS-CoV-2 admitted to ICUs of the Lombardy Region, Italy. JAMA. (2020) 323:1574-81. doi: 10.1001/jama.2020.5394

31. Bhatraju PK, Ghassemieh BJ, Nichols M, Kim R, Jerome KR, Nalla AK, et al. Covid-19 in critically Ill patients in the Seattle Region case series. N Engl J Med. (2020) 382:2012-22. doi: 10.1056/NEJMoa 2004500

32. Fanali G, di Masi A, Trezza V, Marino M, Fasano M, Ascenzi P. Human serum albumin: from bench to bedside. Mol Aspects Med. (2012) 33:20990. doi: 10.1016/j.mam.2011.12.002

33. Yang X, Yu Y, Xu J, Shu H, Xia J, Liu H, et al. Clinical course and outcomes of critically ill patients with SARS-CoV-2 pneumonia in Wuhan, China: a single-centered, retrospective, observational study. Lancet Respir Med. (2020) 8:475-81. doi: 10.1016/S2213-2600(20)30079-5

34. Soeters PB, Wolfe RR, Shenkin A. Hypoalbuminemia: pathogenesis and clinical significance. J Parenter Enteral Nutr. (2019) 43:18193. doi: 10.1002/jpen.1451

35. Kaysen GA. Biochemistry and biomarkers of inflamed patients: why look, what to assess. Clin J Am Soc Nephrol. (2009) 4 (Suppl. 1):S5663. doi: 10.2215/CJN.03090509

36. Mehta P, McAuley DF, Brown M, Sanchez E, Tattersall RS, Manson JJ, et al. COVID-19: consider cytokine storm syndromes and immunosuppression. Lancet Lond Engl. (2020) 395:1033-4. doi: 10.1016/S0140-6736(20) 30628-0

37. Ruan Q, Yang K, Wang W, Jiang L, Song J. Clinical predictors of mortality due to COVID-19 based on an analysis of data of 150 patients from Wuhan, China. Intensive Care Med. (2020) 46:1294-97. doi: 10.1007/s00134-020-06028-z

38. Richardson S, Hirsch JS, Narasimhan M, Crawford JM, McGinn T, Davidson KW, et al. Presenting characteristics, comorbidities, and outcomes among 5700 patients hospitalized with COVID-19 in the New York City Area. JAMA. (2020) 323:2052-9. doi: 10.1001/jama.2020.6775

39. Qin C, Zhou L, Hu Z, Zhang S, Yang S, Tao Y, et al. Dysregulation of immune response in patients with COVID-19 in Wuhan, China. Clin Infect Dis. (2020) 71:762-8. doi: 10.1093/cid/ciaa248

40. Zheng M, Gao Y, Wang G, Song G, Liu S, Sun D, et al. Functional exhaustion of antiviral lymphocytes in COVID-19 patients. Cell Mol Immunol. (2020) 17:533-5. doi: 10.1038/s41423-020-0402-2

41. Chen X, Ling J, Mo P, Zhang Y, Jiang Q, Ma Z, et al. Restoration of leukomonocyte counts is associated with viral clearance in COVID-19 hospitalized patients. medRxiv [Preprint]. (2020). doi: 10.1101/2020.03.03. 20030437 
42. Li D, Chen Y, Liu H, Jia Y, Li F, Wang W, et al. Immune dysfunction leads to mortality and organ injury in patients with COVID-19 in China: insights from ERS-COVID-19 study. Signal Transduct Target Ther. (2020) 5:62. doi: 10.1038/s41392-020-0163-5

43. Cao X. COVID-19: immunopathology and its implications for therapy. Nat Rev Immunol. (2020) 20:269-70. doi: 10.1038/s41577-020-0308-3

44. Pencina MJ, D'Agostino RB, Demler OV. Novel metrics for evaluating improvement in discrimination: net reclassification and integrated discrimination improvement for normal variables and nested models. Stat Med. (2012) 31:101-13. doi: 10.1002/ $\operatorname{sim} .4348$
Conflict of Interest: The authors declare that the research was conducted in the absence of any commercial or financial relationships that could be construed as a potential conflict of interest.

Copyright (C) 2021 Wang, Lin, Wei, Li, Liao, Yuan, Huang, Qin, Geng and Wang. This is an open-access article distributed under the terms of the Creative Commons Attribution License (CC BY). The use, distribution or reproduction in other forums is permitted, provided the original author(s) and the copyright owner(s) are credited and that the original publication in this journal is cited, in accordance with accepted academic practice. No use, distribution or reproduction is permitted which does not comply with these terms. 